\title{
SUPERSONIC DUSTY-GAS FLOWS WITH KNUDSEN EFFECT IN INTERPHASE MOMENTUM EXCHANGE*
}

\author{
WANG Boyi (王柏懿) ${ }^{1, \dagger} \quad$ A. N. OSIPTSOV ${ }^{2} \quad$ L. A. EGOROVA ${ }^{2} \quad$ V. I. SAKHAROV ${ }^{2}$ \\ ${ }^{1}$ (LNM, Institute of Mechanics, Chinese Academy of Sciences, Beijing 100080, China) \\ ${ }^{2}$ (Institute of Mechanics, Moscow State University, Moscow 119899, Russia)
}

\begin{abstract}
On the basis of the two-continuum model of dilute gas-solid suspensions, the dynamic behavior of inertial particles in supersonic dusty-gas flows past a blunt body is studied for moderate Reynolds numbers, when the Knudsen effect in the interphase momentum exchange is significant. The limits of the inertial particle deposition regime in the space of governing parameters are found numerically under the assumption of the slip and free-molecule flow regimes around particles. As a model problem, the flow structure is obtained for a supersonic dusty-gas point-source flow colliding with a hypersonic flow of pure gas. The calculations performed using the full Lagrangian approach for the near-symmetry-axis region and the free-molecular flow regime around the particles reveal a multi-layer structure of the dispersed-phase density with a sharp accumulation of the particles in some thin regions between the bow and termination shock waves.
\end{abstract}

KEY WORDS: gas-solid suspension, supersonic flow, Knudsen effect, particle inertia

\section{INTRODUCTION}

In recent years, mathematical modeling and numerical simulation of supersonic gas-solid two-phase flows have attracted much attention in the fields of aeronautics, astronautics, astrophysics and gasdynamics. Examples of important applications include thermal power equipments and chemical engineering apparatus, as well as certain other technologies (such as cyclone separation, spray coating, powder metallurgy, microchip manufacturing and so on). For a gas-solid suspension (or so-called dusty gas), the gas and solid particles are usually termed as the carrier and dispersed phases, respectively. In general, the presence of particles may result in substantial changes of either local (friction and heat transfer coefficients) or global (shock wave position and flow structure in boundary layer) characteristics. Because of a great difference in the material densities, the particle inertia leads to a relaxation process and momentum/energy exchanges between the two phases. Besides, heavier particles with large inertia may deposit on the body wall while lighter particles with small inertia do not reach the wall but accumulate in the boundary layer. For the deposition case, the surface erosion becomes predominant and the surface heating may be drastically augmented ${ }^{[1,2]}$. For the no-deposition case, the dispersed-phase density may increase in proximity of the wall and the friction/heat transfer to the body also changes noticeably ${ }^{[3,4]}$. Moreover, it is interesting to note that, in many dusty-gas flows, the trajectories of inertial particles may oscillate and then multiple trajectory intersections may appear ${ }^{[5]}$. As a result, the distribution of the dispersed-phase density takes a multi-layer structure. Finally, it is worth to note that the carrier-phase flow around a particle falls into the slip or even free-molecule regime when the particle size decreases to the order of micrometer. When the free streams over a body have moderate Reynolds numbers $\left(10^{2} \leq R e \leq 10^{5}\right)$, the Knudsen number characterizing the flow regime near small particles may be of the order of unity or even attain high

Received 9 July 2002 , revised 16 December 2003

* The project supported by the National Natural Science Foundation of China (90205024), and the Russian Foundation for Basic Research (RFBR grant No. 02-01-00770 and joint RFBR-NSFC grant No. 03-01-39004)

$\dagger$ E-mail: wby@imech.ac.cn 
values. In this case, although the dusty-gas flow past the body (with a characteristic length scale of $1 \mathrm{~m}$ ) is continuous, the Knudsen effect should be taken into account in describing the interphase exchanges. On the basis of numerical simulations, Egorova et al. ${ }^{[6]}$ obtained the critical conditions of the inertial particle deposition for the continuous flow regime around the particles. In this paper, we will find the limits of the deposition regime in the space of non-dimensional governing parameters for the slip and free-molecular flow around the particles. In addition, the flow formed as a result of the interaction of a supersonic pointsource flow of dusty gas with a hypersonic uniform flow of pure gas is studied and the dispersed-phase parameters are calculated using the full Lagrangian approach for the free-molecule regime around the particles.

\section{MODELING OF DILUTE DUSTY-GAS FLOWS}

It is well known that, due to a great diversity of two-phase flows and the great difficulty of experimental investigations, mathematical modeling becomes the main instrument for studying various dusty-gas flows. The most widely used method for the formulation of dusty-gas flow equations is based on the twocontinuum approach ${ }^{[7]}$. However, the inertia effect mentioned above makes the near-wall two-phase flow more complicated and essentially different models are required for the regimes with and without inertial particle deposition. Hence it is necessary to determine the critical conditions of the particle deposition on the frontal surface of a blunt body. In the framework of the dilute dusty-gas model ${ }^{[8]}$, we assume the usual restrictions: (a) the carrier phase is a perfect gas; (b) the particles are non-deformable spheres and their mutual collisions as well as Brownian motion may be ignored; (c) the volume and mass fraction of the dispersed phase is small enough to ignore its effect on the carrier phase. In other words, the dispersed phase is treated as a collisionless "zero-pressure" gas in our model. When the effect of the dispersed phase on the carrier phase is negligible, the computation of the dusty-gas flows may be split into two consecutive steps: the calculation of the carrier-phase parameters and the calculation of the dispersed-phase parameters in the given gas flow field. In this paper, for simplicity, we study only the dynamic problems assuming that the heat exchange between the two phases does not influence the gas-particle force interaction. Besides, the mass exchange between the gas and particles are also ignored due to the absence of chemical reactions and phase changes in our problem.

Generally speaking, in dusty-gas flows, the drag force exerted by the gas on a particle is the main mechanism for particle motion. The expressions for the interphase momentum exchange per particle may be written as follows (below the subscript $s$ refers to the dispersed phase).

(a) In the slip flow regime around the particles ${ }^{[9]}$

$$
\begin{aligned}
& \boldsymbol{f}_{s}^{*}=6 \pi \sigma \mu^{*}\left(\boldsymbol{V}^{*}-\boldsymbol{V}_{s}^{*}\right) D \\
& D=\left(1+\frac{1}{6} R e_{s}^{2 / 3}\right) \Phi\left(M a_{s}, R e_{s}\right) \\
& \Phi=\left(1+\exp \left(-0.427 M a_{s}^{-4.63}-3 R e_{s}^{-0.88}\right)\right) / \varphi \\
& \varphi=1+K n_{s}\left[2.57+0.68 \exp \left(-1.86 / K n_{s}\right)\right]
\end{aligned}
$$

(b) In the free-molecular flow regime around the particles ${ }^{[10]}$

$$
\begin{aligned}
& \boldsymbol{f}_{s}^{*}=\frac{\pi}{2} \sigma^{2} \rho^{*}\left|\boldsymbol{V}^{*}-\boldsymbol{V}_{s}^{*}\right|\left(\boldsymbol{V}^{*}-\boldsymbol{V}_{s}^{*}\right) D_{r} \\
& D_{r}=\frac{1}{S^{3}}\left[\frac{4 S^{4}+4 S^{2}-1}{2 S} \operatorname{erf}(S)+\right. \\
& \left.\frac{2 S^{2}+1}{\sqrt{\pi}} \exp \left(-S^{2}\right)\right] \\
& S=\sqrt{\frac{\gamma}{2} M a_{s}} \\
& \operatorname{erf}(S)=\frac{2}{\sqrt{\pi}} \int_{0}^{s} \exp \left(-t^{2}\right) \mathrm{d} t
\end{aligned}
$$

In Eqs.(1) and (2), $\rho^{*}, \boldsymbol{V}^{*}$ and $\sigma$ are the phase density, the velocity vector and the particle radius, and $\mu^{*}$ is the gas viscosity in the power-law relation with temperature: $\mu^{*} / \mu_{0}^{*}=\left(T^{*} / T_{0}^{*}\right)^{\omega}$. Here, the superscript ${ }^{*}$ denotes dimensional variables to distinguish them from the corresponding non-dimensional ones where necessary. In the expressions above, there are three similarity parameters characterizing the gas flow around the particles: (a) Reynolds number $\boldsymbol{R e}_{s}=2 \sigma\left|\boldsymbol{V}^{*}-\boldsymbol{V}_{s}^{*}\right| \rho^{*} / \mu^{*}$; (b) Mach number $M a_{s}=\left|\boldsymbol{V}^{*}-\boldsymbol{V}_{s}^{*}\right| / a^{*} ;$ (c) Knudsen number $K n_{s}=$ $l / 2 \sigma \approx 1.255 \sqrt{\gamma} M a_{s} / \operatorname{Re} e_{s}$. Here, $a^{*}$ is the speed of sound in gas, $l$ the gas molecular free path, and $\gamma$ the gas specific heat ratio. Obviously, expression (1) is a modified form of the usual drag formula for the continuous flow around the particles. The correction function $\varphi$ takes into account the finiteness of the particle Knudsen number, when the flow around the particles falls into the slip regime. This expression is valid 
only for this case of small deviation from the continuous flow regime. As $K n_{s} \rightarrow 0$, the flow around the particles becomes continuous and we have $\varphi=1$. As $K n_{s} \rightarrow \infty$, the flow around the particles becomes free-molecular and we have expression (2) for representing the particle drag. It should be noted that the dusty-gas flows over bodies under consideration are still continuous although the gas flow around the particles may fall into the slip or free-molecule regime.

In order to determine the limits of the inertial particle deposition regime, consider a steady uniform supersonic flow of viscous dusty gas past a spherical body of radius $R_{0}^{*}$. The carrier-phase flow parameters are found from a numerical solution of the NavierStokes equations on a non-uniform grid (see Ref.[6] for details). For generality, all physical variables are normalized using the length $R_{0}^{*}$ and the corresponding free-stream parameters (denoted by the subscript $\infty$ ) in our mathematical formulation. In the limiting situation of the free-molecular flow around the particles $\left(K n_{s}=\infty\right)$, the particle motion equation can be written in the following non-dimensional form

$$
\begin{aligned}
& \frac{\mathrm{d} \boldsymbol{V}_{s}}{\mathrm{~d} t}=A \rho D_{r}(S)\left|\boldsymbol{V}-\boldsymbol{V}_{s}\right|\left(\boldsymbol{V}-\boldsymbol{V}_{s}\right) \\
& A=\frac{1}{24}\left(\frac{\gamma-1}{\gamma+1}\right) \beta R e_{s 0}
\end{aligned}
$$

where $\beta=6 \pi \sigma \mu_{c}^{*} R_{0}^{*} / m U_{\infty}$ is the particle inertia parameter (with $m$ being the particle mass) and $R e_{s 0}=$ $2 \sigma \rho_{c}^{*} U_{\infty} / \mu_{c}^{*}$ is the particle Reynolds number based on the adiabatic stagnation parameters (denoted by the subscript $c$ ). Through a numerical calculation, the critical value of $A$ (corresponding to the inertial deposition limit) can be found from the following condition: the particle velocity at the stagnation point of the body is zero within the accuracy of the twelfth decimal place. The limits for the slip regime are easily obtained by using the same method. The calculated limits are plotted in the plane $\left(\beta_{0}, R b\right)$ so that it is convenient to compare them with the inertial deposition limits for the continuous flow around the particles. Here $\beta_{0}=\beta \mu_{1} / u_{1} \varphi_{1}$ is the scaled particle inertia parameter (the subscript 1 refers to the non-dimensional parameter at the inviscid-flow stagnation point and $u_{1}$ is the modules of the gas velocity gradient at the stagnation point) and $R b=R e_{s 0}^{2 / 3} / 6$ is a parameter characterizing the particle drag deviation from the linear law. The results obtained for the Mach number of the flow past a sphere $M a=6$ are presented for different Reynolds numbers (based on the free-stream parameters and the body radius)
$R e=10^{5}, 10^{4}, 10^{3}, 10^{2}$ (corresponding to curves $1 \sim 4$, respectively) in Fig.1, where the regions above the curves are the zones without particle deposition on the frontal surface of the body immersed in the supersonic dusty-gas flow. These results indicate: (a) For large Knudsen numbers $\left(K n_{s} \rightarrow \infty\right)$, the critical values of the particle inertia parameter for the inertial particle deposition regime are greater than those in the continuous flow case. (b) For moderate Knudsen numbers $\left(K n_{s} \approx 1\right)$, the limits obtained by using Eqs.(1) and (2) coincide in the order of magnitude. Here, the relation between $K n_{s}$ and $R b$, which are both based on the particle diameter, is given in Table 1.

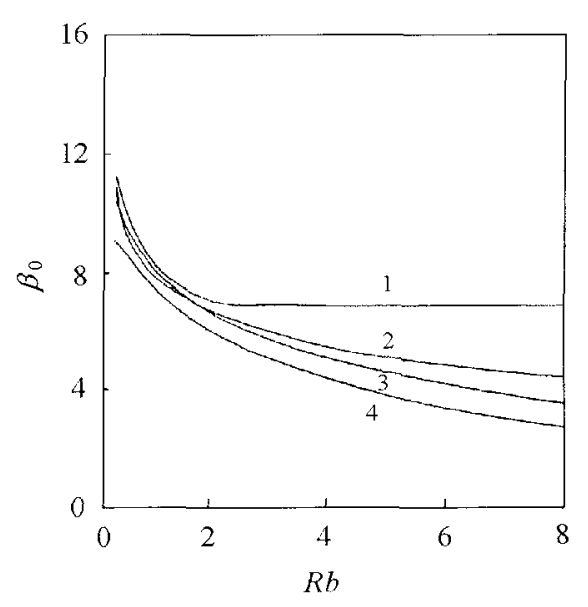

(a) Slip flow case

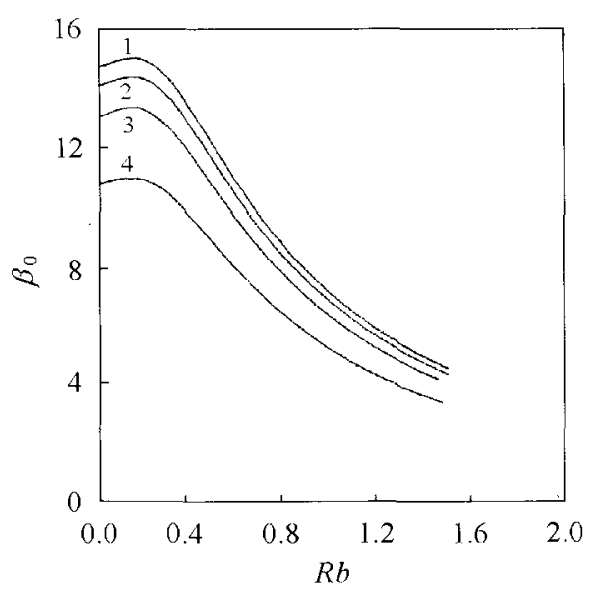

(b) Free-molecular flow case

Fig.1 Limits of the inertial deposition regime. Curves $1 \sim 4$ correspond to $R e=10^{5}, 10^{4}, 10^{3}$ and $10^{2}$

Table 1

\begin{tabular}{cccccccc}
\hline$K n_{s}$ & 0 & 0.01 & 0.1 & 1.0 & 10 & 100 & $\infty$ \\
\hline$R b$ & $\infty$ & 7.992 & 1.722 & 0.371 & 0.0172 & 0.0799 & 0 \\
\hline
\end{tabular}




\section{GOVERNING EQUATIONS FOR DIS- PERSED PHASE}

In most practical applications, gas-solid twophase systems are dilute and the effect of the particles on the gas is negligible. The basic equations governing the carrier-phase flow are the Navier-Stokes equations, which are not reproduced here to save space. Various numerical methods are available to solve the pure-gas flows, but, in this paper, of concern is how to determine the dispersed-phase parameters (including the density), especially, in case of possible intersections of particle trajectories. As an example, we consider a steady flow formed as the result of the interaction of a supersonic dusty-gas point source with a hypersonic pure-gas flow, assuming that the colliding gases have the same thermodynamic properties. This model problem has its applications in interpreting astrophysical phenomena (for instance, experimental data on comet atmospheres), in investigating aerospace engineering problems (such as underexpanded nozzle jets in an opposite flow or mass injection from the nose of a high-speed vehicle), as well as civil industrial technologies (for example, drying powder materials). For the problem under consideration, a termination shock wave (termed as shock 1) appears in the flow from the source while a bow shock wave appears in the oncoming flow. The inner and outer shock layers are separated by a contact surface. As usual, $U_{\infty}$ and $R_{0}^{*}$ refer to the free-stream velocity and the curvature radius of the shock 1 at the stagnation point. Besides, we assume that the carrier phase from the source reaches its maximum velocity prior to the shock 1, where the gas and particles are in equilibrium. Obviously, the particle inertia will play some role behind the shock 1 . The gas flow parameters between the two shock waves in the neighborhood of the symmetry axis can be obtained from the hypersonic gasdynamics ${ }^{[1]}$. For describing the dynamic behavior of particles in dilute two-phase systems, the Lagrangian approach is widely used, especially for those dusty-gas flows, in which particle trajectories intersect and the usual Eulerian approach cannot be employed for the dispersed phase, since the uniqueness of the particle parameters is violated. However, the traditional Lagrangian approach requires tracing a large number of particles. We employ the novel method developed by Osiptsov ${ }^{[12]}$, which makes it possible to determine all the dispersed-phase parameters (including the density) along the chosen particle trajectories. Due to a considerable reduction of the "cost" of computations, this method becomes more and more pop- ular (see Ref.[13] for example).

For the mathematical formulation of the problem, the coordinate system $(x, y)$ fixed to shock 1 is introduced with the origin at the stagnation point. In the Lagrange coordinates $x_{0}$ (non-dimensional initial coordinate of the particle trajectory on shock 1) and $t$ (non-dimensional time of the particle motion along the chosen trajectory), the equations of motion for the dispersed-phase take the form

$$
\begin{aligned}
& \frac{\mathrm{d} u_{s}\left(x_{0}, t\right)}{\mathrm{d} t}=f_{s x}-\frac{u_{s} v_{s}}{R+y_{s}} \\
& \frac{\mathrm{d} v_{s}\left(x_{0}, t\right)}{\mathrm{d} t}=f_{s y}-\frac{u_{s}^{2}}{R+y_{s}}
\end{aligned}
$$

In addition, there are two kinematic relations

$$
\frac{\mathrm{d} x_{s}}{\mathrm{~d} t}=\frac{u_{s}}{1+y_{s} / R} \quad \frac{\mathrm{d} y_{s}}{\mathrm{~d} t}=v_{s}
$$

where $x_{s}$ and $y_{s}$ are the coordinates of the particle position at time $t, u_{s}$ and $v_{s}$ are the longitudinal and normal velocities, $f_{s x}$ and $f_{s y}$ are the longitudinal and normal components of the drag force, and $R(x)$ is the curvature radius of shock 1. Equations (4) and (5) have the form of ordinary differential equations along the particle trajectories and are convenient for the numerical solution. The non-dimensional form of the drag force in Eq.(4) can be written as follows:

(a) In the slip regime for the flow around the particles

$$
\begin{aligned}
& f_{s}=\beta \mu D\left(\boldsymbol{V}-\boldsymbol{V}_{s}\right) \\
& \beta=6 \pi \sigma \mu_{c}^{*} R_{0}^{*} / m U_{\infty}
\end{aligned}
$$

(b) In the free-molecule regime for the flow around the particles

$$
\begin{aligned}
& \boldsymbol{f}_{s}=(3 \sqrt{\pi} / 16) \beta_{r} \rho \mu D_{r}\left(\boldsymbol{V}-\boldsymbol{V}_{s}\right) \\
& \beta_{r}=8 \sqrt{\pi(\gamma-1) / \gamma} \rho_{\infty}^{*} \sigma^{2} R_{0}^{*} / 3 m
\end{aligned}
$$

where $\beta=R_{0}^{*} / l_{v}$ and $\beta_{r}=R_{0}^{*} / l_{v r}$ are the inertia parameter, respectively, for the slip and free-molecule regimes $\left(l_{v}\right.$ and $l_{v r}$ are the corresponding characteristic length of the phase velocity relaxation). Starting from the continuity equation in the Euler coordinates and taking into account the boundary conditions on shock 1 , the continuity equation for the dispersed phase in the Lagrange coordinates can be written as

$$
\begin{aligned}
& \frac{\rho_{s}\left(x_{0}, 0\right)}{\rho_{s}\left(x_{0}, t\right)}=\frac{r_{e}\left(x_{s}, y_{s}\right)}{r_{w}\left(x_{0}\right)} J \\
& J=\left(1+\frac{y_{s}}{R}\right) \frac{v_{s}\left(x_{0}, t\right)}{v_{s}\left(x_{0}, 0\right)} \frac{\partial x_{s}}{\partial x_{0}}-\frac{u_{s}\left(x_{0}, t\right)}{v_{s}\left(x_{0}, 0\right)} \frac{\partial y_{s}}{\partial x_{0}}
\end{aligned}
$$


where $r_{e}$ and $r_{w}$ are the distances from the symmetry axis to the flow-field point and to the surface of shock 1 , respectively. This equation contains the components of the Jacobian of the transformation from the Euler to Lagrange coordinates and gives the relation between the initial and current values of the dispersed-phase density along a fixed particle trajectory $\left(x_{s}\left(x_{0}, t\right), y_{s}\left(x_{0}, t\right)\right)$. In Osiptsov method, four auxiliary functions and four ordinary differential equations for these functions are introduced and the density of the dispersed phase at any instant of time on the given trajectory can be easily obtained (see Refs. $[12,13]$ for details).

\section{NUMERICAL RESULTS}

By using the Kutta-Merson method, the numerical computation of the problems (4), (5) and (8) was performed for the near-symmetry-axis region $(0 \leq x \leq 0.4)$. The thermodynamic parameters of the colliding gases are $\gamma=1.4, P r=0.75$ and $\omega=0.5$. According to Osiptsov method, we calculated first the trajectories of the particle continua, which are located initially at the surface of shock 1 and then the density development along each particle trajectory. The calculations demonstrate that, depending on the inertia parameter, two kinds of particle motion may occur: (a) following the gas streamlines, the light particles travel in the inner shock layer monotonically approaching the contact surface; (b) deviating from the gas streamlines, the heavy particles oscillate and approach the contact surface, intersecting it several times. In the latter case, near the envelopes of the particle trajectories, the dispersed-phase density becomes infinite since the normal velocity of the particles vanishes at the turn points of their trajectories. In the neighborhood of the contact surface, the particles also accumulate but this effect here is weaker than that in the envelope region. Between the two envelopes, multiple intersections of the particle trajectories occur. This presents considerable computation difficulties with regard to non-uniqueness of the dispersed-phase parameters. The multiple intersection results in a sharp stratification of the dispersed phase in the shock layers. In order to illustrate this phenomenon, the particle density distribution was calculated for fairly inertial particles $\left(\beta_{r}=0.15\right)$ in the free-molecule regime. The particle density profiles of three different positions $x=0,0.1$ and 0.4 are plotted in Fig.2, where only three types of particle continua are considered. To be specific, they intersect the line $x=$ const: (a) before the first trajectory turn of the first continua (see curve 1 for $x=0,0.1,0.4$ ); (b) after the first and before the second trajectory turn of the second continua (see curve 2 for $x=0,0.1$ and 4 for $x=0.4$ ); (c) after the second and before the third trajectory turn of the third continua (see curve 3 for $x=0$ and 5 for $x=0.4$ ). From the calculations, the contributions of the other particle continua to the total density may be ignored. To find the total particle density at given Euler coordinates $(x, y)$, we must sum up the densities of each particle continuum there. It is clear that the dispersed phase has a multilayer structure with alternating low- and high-density layers and it tends to infinity near the envelopes. The calculated number of the accumulation layers is shown in Fig.3. Accumulation zones of a given number exist

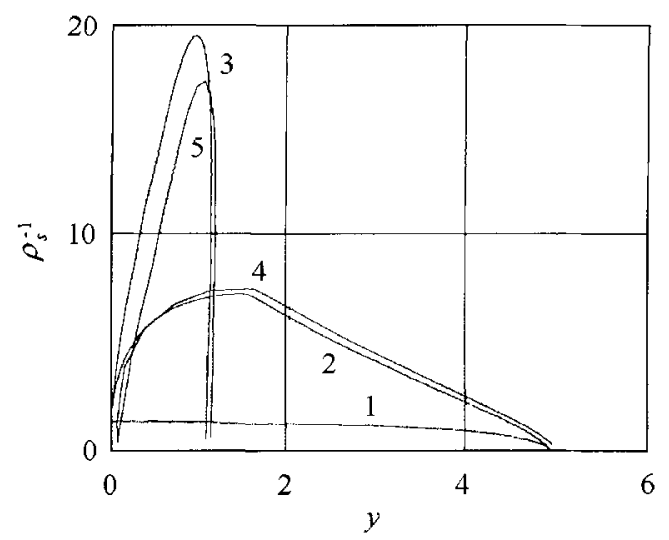

Fig.2 Density of the dispersed phase in freemolecule regime around the particles: Curve 1 corresponds the first continuum at $x=0,0.1,0.4$; Curves 2 and 4 correspond the second continuum at $x=0,0.1$ and $x=0.4$; Curves 3 and 5 correspond the third continuum at $x=0$ and $x=0.4$

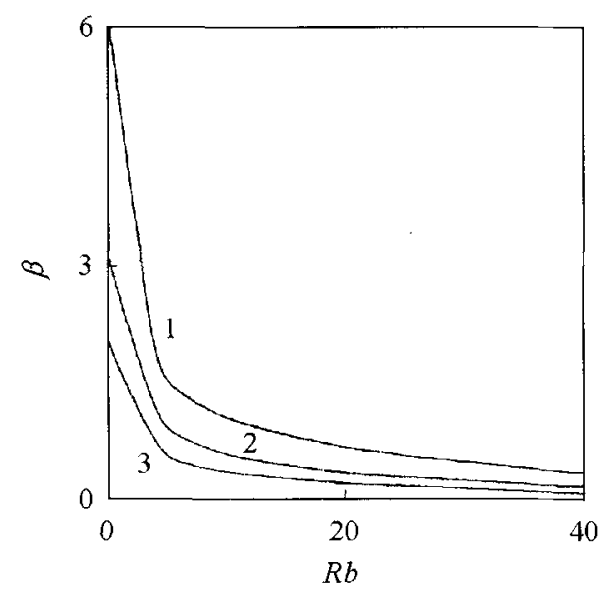

(a) Slip regime

Fig.3 Regions of existence of particle accumulation zones. Curves $1 \sim 3$ correspond to the number of the particle accumulation zones $n=1,2$ and 3 


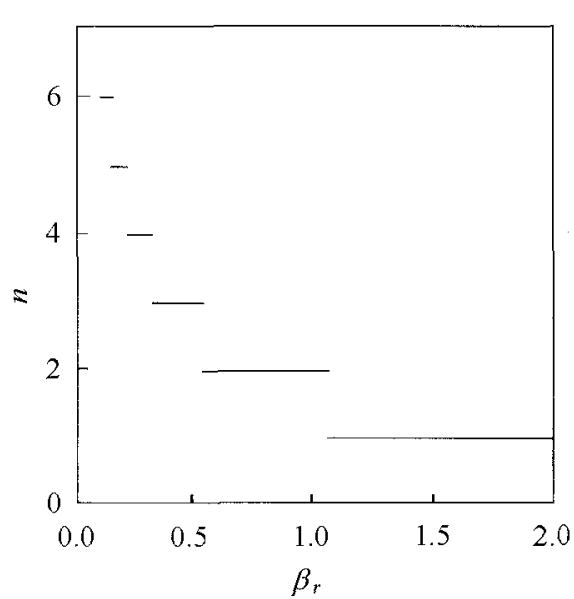

(b) Free-molecular regime

Fig.3 Regions of existence of particle accumulation zones. Curves $1 \sim 3$ correspond to the number of the particle accumulation zones $n=1,2$ and 3 (continued)

when the parameters fall in the region above the corresponding curve. In the slip regime, the zone number $n$ depends on two parameters $(R b$ and $\beta)$ and, due to the Knudsen effect, the distance between the zone boundaries sharply increases with decreasing $R b$ (see Fig.3(a)). On the contrary, in the free-molecule regime, the number $n$ depends on single parameter $\left(\beta_{r}\right)$. The calculations indicate that, due to the inertia effect, the accumulation zone number increases and the zone width becomes narrower with decrease of $\beta_{r}$ (see Fig.3(b)).

\section{CONCLUSIONS}

On the basis of the two-continuum model, the dynamic behavior of inertial particles in supersonic flows of a dilute gas-solid suspension was studied for the Reynolds number of the flow around the body ranging between $10^{5}$ and $10^{2}$. The limits of the inertial particle deposition regime in the space of governing parameters were found numerically under the assumption of the slip and free-molecule flow regimes around the particles. Due to the Knudsen effect, the critical value of the particle inertia parameter for deposition becomes higher than that in the continuousflow case. In the region of moderate particle Knudsen numbers, the limits obtained for the slip and freemolecule regimes coincide in the order of magnitude. For the model problem of a supersonic point source flow of the dusty gas colliding with a hypersonic flow of the pure gas in the free-molecule regime around the particles, the calculations reveal the formation of a stratified structure in the density distribution of the dispersed phase and the appearance of particle accumulation near the trajectory envelopes and the contact surface. The number of the high-density layers increases with increase of the particle inertia. The Knudsen effect as well as the inertia effect plays a significant role in the modeling of supersonic gas-solid two-phase flows.

\section{REFERENCES}

1 Dunbar LE, Courtney JF, McMillen LD. Heating augmentation in erosive hypersonic environments. AIAA $J, 1975,13(2): 908 \sim 912$

2 Humphrey JAC. Fluid motion in erosion by solid particle impact. Int I Heat and Fluid Flow, 1990, 11(3): $170 \sim 195$

3 Osiptsov AN, Shapiro EG. Effect of fine particles on the boundary layer structure in hypersonic flow past a blunt body (in Russian). Izv Akad Nauk SSSR, Mekh Zhidk Gaza, 1986, (5): 55 62

4 Osiptsov AN, Rozin AV, Wang BY. Non-stationary effects in hypersonic nonuniform dusty-gas flow past a blunt body. Acta Mechanica Sinica, 1999, 15(3): $201 \sim 214$

5 Osiptsov AN, Shapiro EG. Two-phase mass injection on the nose of a blunt body in a hypersonic gas flow (in Russian). Izv Ross Akad Nauk Mekh Zhidk Gaza, 1992, (4): $60 \sim 66$

6 Egorova LA, Osiptsov AN, Sakharov VI. On the limits of the inertial particle deposition regime and heat transfer in a supersonic dusty-gas flow past bodies (in Russian). Izv Ross Akad Nauk Mekh Zhidk Gaza, 2001, (6): 111 124

7 Nigmalulin RI. Dynamics of Multiphase Media. New York: Hemisphere, 1990

8 Marble F. Dynamics of dusty gases. Ann Rev Fluid Mech, 1970, 2: 397 446

9 Carlson DJ, Hoglund RF. Particle drag and heat transfer in rocket nozzles. AIAA J, 1964, 2(11): 1980 1984

10 Bondarev EN et al. Aerohydromachanics (in Russian). Moscow: Mashinostroenie, 1993

11 Lunev VV. Hypersonic Aerodynamics (in Russian). Moscow: Mashinostroenie, 1975

12 Osiptsov AN, et al. Novel Lagrangian method for calculating the particle concentration in dusty-gas flows with intersecting particle trajectories. In: Zhou LX, Li XF eds. Multiphase Fluid, Nom-Newtonian Fluid and Physico-Chemical Fluid Flows, Vol 2. International Academic Publisher, 1997. 65 72

13 Healy DP, Young JB. Calculation of inertial particle transport using the Osiptsov Lagrangian method. In: Proc. 4th Intern Conf Multiphase Flow, ICMF'2001, New Orleans, USA, 2001. CD: 1 12 